\title{
Recherches Interdisciplinaires Sur Les Sites Funéraires Des Régions De La Bagoué Et Du Poro En Côte d'Ivoire
}

\author{
Kiénon-Kaboré Timpoko Hélène (Maître de Conférences) \\ Bouadi Kouadio René (Assistant) \\ Thia-Assouan Aline (Maître-assistante) \\ Atta Kouamé (Maître-assistant) \\ Kouassi Firmin (Maître-assistant) \\ Coulibaly Drissa (Assistant) \\ Dappa Joëlle (Doctorant) \\ Tui Lah Louis (Doctorant) \\ Dougan Salia (Doctorant) \\ Kazio Didjé Jacques (Doctorant) \\ Université Félix Houphouët-Boigny, Cocody, Institut des Sciences \\ Anthropologiques de Développment (ISAD), Côte d'Ivoire
}

doi: 10.19044/esj.2017.v13n27p318 URL:http://dx.doi.org/10.19044/esj.2017.v13n27p318

\begin{abstract}
Within the framework of the project: "contribution of the social sciences and the pharmacology to the process of the sustainable development and struggle against poverty in the region of Poro and Bagoué", a first campaign of archaeological and anthropological research has been undertaken in the localities of Tongon and Poungbè in the region of Bagoue, located in the north of Côte d'Ivoire, approximately $50 \mathrm{~km}$ in the north of Korhogo city; administrative center of the region of Poro. This mission, which enters into a multidisciplinary approach, deals with the funeral sites of Nawavogo and Daovogo that is presented by traditionnists as former metallurgists graves to whom we attribute the sites of restriction of the iron, extraction of the ore, and the habitat in the locality of Nawavogo and Poungbè. The first results based on the topographic analysis of sites, the preliminary study of the ceramic, and the two excavated graves show many specific funeral practices and a technical know-how of the populations which have occupied these spaces. The scientific, cultural and patrimonial value of these data has already explained all the interest to make the study of this region works deeper.
\end{abstract}


Keywords: Archaeological - anthropological - sites - funeral - graves ceramic

\section{Resume :}

Dans le cadre du projet : "Contribution des sciences sociales et de la pharmacologie au processus de développement durable et de la lutte contre la pauvreté dans la région du Poro et de la Bagoué», une première campagne de recherche archéologique et anthropologique a été entreprise dans les localités de Tongon et de Poungbè dans la région de la Bagoué, située au Nord de la Côte d'Ivoire à environ $50 \mathrm{~km}$ au Nord de la ville de Korhogo chef-lieu de la région du Poro. Cette mission qui s'inscrit dans une approche interdisciplinaire, aborde les sites funéraires de Nawavogo et de Daovogo présentés par les traditionnistes comme des sépultures d'anciens métallurgistes à qui l'on attribue les sites d'extraction, de réduction du minerai et d'habitat dans la localité de Nawavogo et de Poungbè. Les premiers résultats basés sur l'analyse topographique des sites, l'étude préliminaire de la céramique et de deux sépultures fouillées, montrent bien des pratiques funéraires spécifiques et un savoir-faire technique des populations qui ont occupées ces espaces. La valeur scientifique, culturelle et patrimoniale de ces données traduit déjà tout l'intérêt à approfondir les travaux dans cette région.

Mots clés : Archéologique - anthropologique - sites funéraires - sépultures - céramique

\section{Introduction}

En phase avec son programme d'activités, le groupe de recherches interdisciplinaires, Archéologie, Technique et Patrimoine (A.T.Pa.), .) a entrepris une mission de recherche dans les localités de Tongon et Poungbè du 17 mars au 04 avril 2017. Neuf chercheurs issus de divers domaines, ont pris part à cette étude. Ce sont cinq Archéologues ${ }^{1}$, un Paléoanthropologue ${ }^{2}$, un Bioanthropologue ${ }^{3}$ et deux Socioanthropologues ${ }^{4}$.

\footnotetext{
1 - Les cinq Archéologues sont : le Dr Kiénon-Kaboré Hélène, responsable du projet et du groupe de recherche, le Docteur Bouadi Kouadio René, les Doctorants Kazio Djidjé Jacques, Dapa Marie-Joelle et Tui La Louis.

2 - Le Docteur Kouassi Firmin étant absent, le Docteur Coulibaly Siaka a intervenu pour le compte de la Paléoanthropologie.

3 - Le Docteur Kouamé Atta a pris part à cette mission en tant que Bioanthropologue.

4 - La socioanthropologie a été représentée par le Docteur Tia Aline et le Doctorant Dougan Salia.
} 
Pour cette première mission, il était question d'identifier les sites funéraires dont l'étude permettrait de comprendre le mode de vie des populations antérieures de cette région. Des recherches sur la métallurgie ancienne du fer ont été entreprises dans cette localité et ont révélé une grande richesse en site de la sidérurgie directe. Les traditionnistes attribuent des sépultures, spécifiques par leur localisation au niveau du relief, aux métallurgistes $\mathrm{du}$ fer jadis installés dans cette région. De ce fait, les sites funéraires de Nawavogo et de Daovogo ont suscité une attention particulière de la part des chercheurs. Ils ont fait l'objet d'une prospection intensive, de relevés topographiques et de fouilles archéologiques. Au cours de cette mission de recherche deux sépultures ont été fouillées sur le site funéraire de Nawavogo ${ }^{5}$. L'objectif de l'approche archéologique dans cette étude interdisciplinaire est de comprendre, d'abord l'aire de répartition de ces nécropoles situées sur des reliefs spécifiques, ensuite l'organisation spatiale des sites, et enfin les cultures et la répartition chronoculturelle de l'ensemble des sites funéraires, dans le but de caractériser les cultures et les peuples auteurs de celles-ci.

Pour cette mission de recherche, l'objectif principal est de comprendre l'organisation spatiale des deux nécropoles, ainsi que la culture en présence.

Pour rendre compte de cette première approche, le présent article s'articule autour de trois points :

- $\quad$ le cadre de l'étude ;

- l'approche méthodologique;

- les premiers résultats de terrain.

\section{Cadre de l'étude}

Les vestiges archéologiques divers découverts dans la zone depuis 2010 sont la preuve que des activités se sont développées à une période donnée dans cette localité. Des populations y ont vécu. Le cadre physique constitue le plus souvent un atout essentiel à l'installation des hommes et à la mise en place de certaines cultures.

\section{Le cadre physique et humain}

Située au Nord de la Côte d'Ivoire, précisément entre le $10^{\text {ème }}$ parallèle au Sud et le $9^{\text {ème }}$ parallèle à l'Est, à l'Ouest entre les $6^{\text {ème }}$ et $5^{\text {ème }}$ degrés de longitude, la zone de Korhogo-M'Bengué selon le découpage administratif de 2012, est localisée dans les régions du Poro et de la Bagoué. Elle livre une diversité de sites archéologiques et une richesse culturelle

${ }^{5}$ Les fouilles archéologiques ont été entamées sur deux sépultures du site de Nawavogo mais ne sont pas achevées au niveau de la tombe2. Elles seront poursuivies au cours des prochaines missions. Nous présentons dans cet article, des résultats préliminaires. 
indéniable. Le peuplement ancien et actuel aurait été favorisé par les dispositions de l'environnement naturel propice à l'installation de l'homme. La végétation appartient au secteur sub-soudanais, caractéristique de la savane arborée, de la forêt galerie et de la savane dégradée. Le climat est de type tropical subhumide caractérisé par une saison sèche (Novembre-Mai) et une saison pluvieuse (Juin-Octobre). Les activités de la métallurgie ancienne du fer, dont divers restes archéologiques ont été identifiés sur ces zones, sont menées en tenant compte de ces différentes saisons et climats. La végétation est typique des sols nus qui sont les zones de prédilection des cuirasses. Elle est riche et variée et renferme une panoplie de plantes qui intervient dans différents domaines de la vie quotidienne, économique, culturelle, cultuelle etc. (Avy S.K. et al, 2006 : 208). Les formations géologiques sont d'âge protérozoïque et les roches appartiennent au complexe éburnéen. Cette région est caractérisée par une succession de bandes de roches schisteuses et de roches magmatiques et plutoniques. (Beaudou A.G, Sayol R., 1988 : 7.) Ces formations sont riches en minerai de diverses sortes qui ont favorisé surtout la métallurgie ancienne du fer, une activité très importante dans la vie de ces populations dans la région. Les vestiges archéologiques sont des témoins de ces activités. La richesse de ce patrimoine a été l'une des raisons de la mise en place de ce projet interdisciplinaire.

Au niveau humain, issus du groupe Gur, les Sénoufo occupants actuels de cet espace, se nomment «sénarbélé» qui signifie agriculteurs, et « sénar» marquant la langue et «bélé » le pluriel (Duc P., 2012 :26). Les Sénoufo sont ceux qui parlent le «sénar». "Sénarbélé » est aussi un marqueur d'identité. Ils occupent un vaste espace de l'Afrique subsaharienne à l'Ouest et sont à cheval sur le Burkina Faso, le Mali et la Côte d'Ivoire. Leurs ancêtres sont les Falafala ou Pallaka ou encore les Myoro (Gonnin et Allou, 2006 : 48). Ce groupe ethnique comprend trois grands groupes, selon le pays d'implantation; mais plusieurs éléments révèlent une unité fondamentale entre eux : la langue de base, la pensée religieuse, les scarifications autrefois pratiquées, la définition de la famille, la primauté du groupe sur l'individu, le pacifisme et l'importance primordiale du travail de la terre. Ils sont localisés dans le Nord de la Côte d'Ivoire et qualifiés par certains auteurs de peuples autochtones (Gonnin et Allou, 2006 : 47).

\section{Historique des recherches}

Depuis 2010, des missions de recherches sont entreprises dans la zone de Korhogo et de M'Bengué. Elles ont permis de mettre au jour plusieurs sites archéologiques à Flanakaha, Koni, Koffiplé, Zemongokaha, Dokélédougou (Nawavogo) Poungbè et Kantara (Kiénon-Kaboré, 2012 : 153). Des sites d'habitat ont été identifiés à Poungbè, à Nawavogo sur plusieurs secteurs et le plus souvent proches de sites de métallurgie ancienne 
du fer composés de fourneaux de réduction du fer, de grands amas de scories de fer, associés aux structures en cercles de pierres faisant à priori penser à des bases de constructions circulaires, de puits d'extraction du minerai de fer et des sites funéraires dont celui de Nawavogo découvert en 2011. (cf. Carte des sites archéologiques). En marge de ces recherches archéologiques, des investigations avaient été faites sur le patrimoine culturel et artisanal qui présente une diversité et une richesse importantes. Cependant, on remarque une dichotomie entre les richesses culturelles, archéologiques, pharmacologiques et la pauvreté des populations. Ce projet interdisciplinaire intitulé "Contribution des sciences sociales et de la pharmacologie au processus du développement durable et de la lutte contre la pauvreté dans la région du Poro et de la Bagoué. " vise à aborder les aspects archéologiques, historiques, anthropologiques et pharmacologiques afin de contribuer à une politique de lutte contre la pauvreté dans une perspective de développement durable. En effet, après 6 ans d'investigations archéologiques dans les régions du Poro et de la Bagoué, nous nous sommes rendu compte des richesses diverses dont le patrimoine technique (l'architecture, la céramique, la teinture etc.), archéologique et pharmacologique qui tendent à disparaitre. L'appropriation de ces savoirs et techniques et la connaissance des modes de vie des populations anciennes dans la lutte contre la pauvreté est possible si l'on met en place une politique adéquate et adaptée aux réalités socioculturelles; d'où l'importance de la synergie entre ces disciplines. La question fondamentale qui se pose est : comment contribuer au développement durable de la région du Poro et de la Bagoué en mettant à contribution les sciences sociales et pharmacologiques? En effet, la région est très riche en plantes médicinales qui influe sur la vie des populations actuelles.

Pour cette mission du 17 Mars au 4 Avril 2017, nous avons mis l'accent sur l'approche archéologique tout en faisant des recherches prospectives pour les autres disciplines impliquées. 


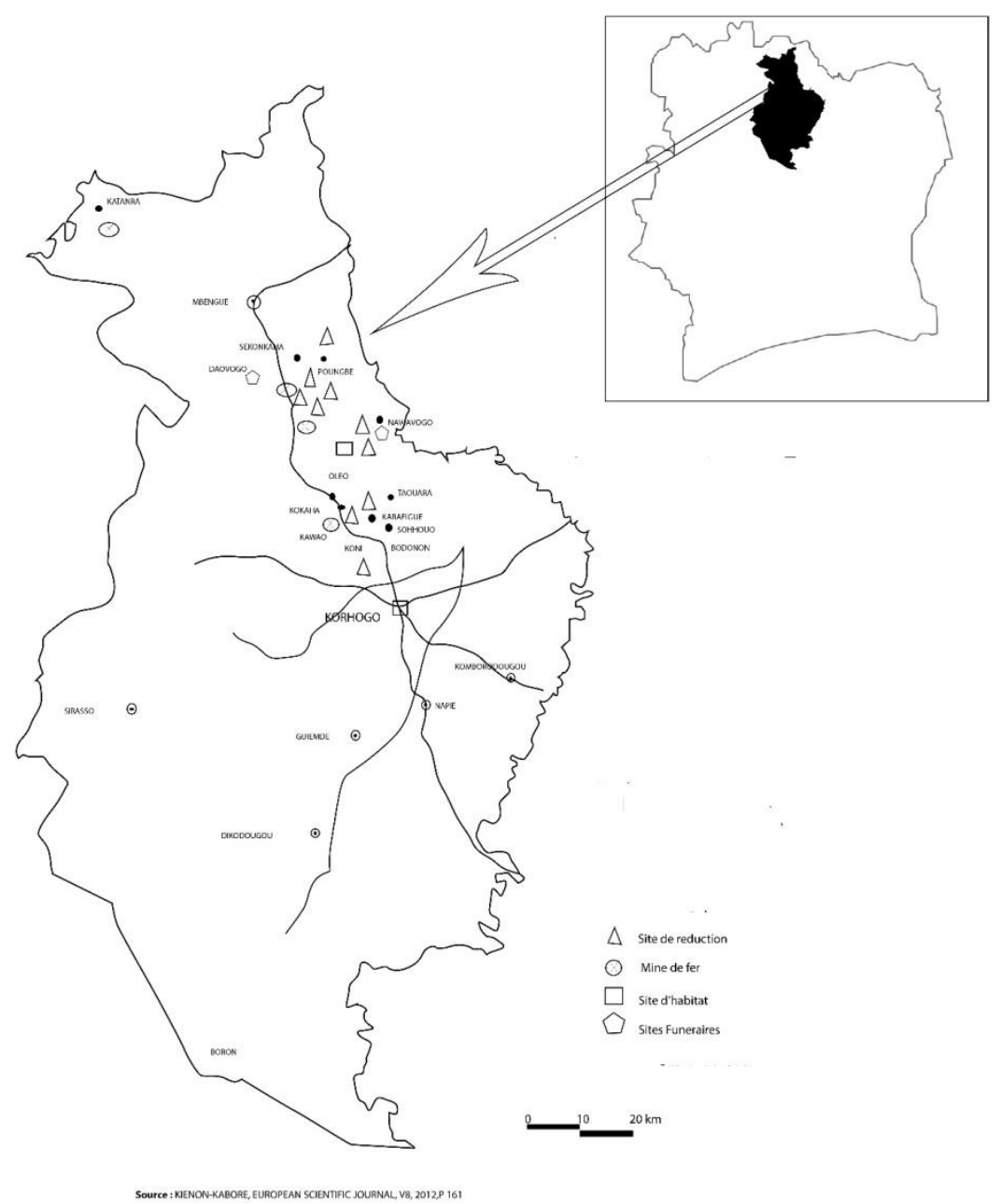

\section{Approche méthodologique}

Après une recherche documentaire qui a permis d'avoir des données significatives, nous avons appliqué, respectivement une approche de terrain basée sur l'enquête orale auprès des traditionnistes, la prospection archéologique afin de localiser et déterminer les limites des sites, les levées topographiques pour cerner l'organisation spatiale des sites funéraires et enfin les fouilles archéologiques pour comprendre les sépultures et la culture des peuples jadis installés sur cet espace. Tout cela s'est fait dans une 
approche interdisciplinaire pour répondre aux objectifs de la recherche. Cependant pour cet article, nous abordons les aspects archéologiques

\section{Enquête orale et prospection archéologique}

L'enquête orale et la prospection archéologique ont souvent été abordées de façon complémentaire pour la localisation des sites, la compréhension de l'histoire de l'installation des populations et des cultures des populations récentes et anciennes.

\section{L'enquête orale}

Une enquête orale a été réalisée dans le village de Poungbè et au campement Nawavogo. Celle-ci visait à recueillir des informations sur l'histoire du peuplement, les activités artisanales et les techniques anciennes, notamment la réduction traditionnelle du minerai de fer dans cette localité. Compte tenu des nombreux sites de la métallurgie ancienne du fer répertoriés dans la région et le lien établi par les traditionnistes entre ces vestiges et les sites funéraires, l'enquête a été orientée sur les pratiques liées à cette activité et celles liées aux rites funéraires. Ceci afin d'avoir une meilleure approche culturelle de ces populations et de comprendre les sites funéraires anciens localisés dans la zone par une comparaison des données culturelles, archéologiques et anthropologiques. Cette enquête devrait compléter les informations recueillies lors des précédentes missions entreprises depuis 2010.

Ainsi, des personnes ressources ont été interrogées sur plusieurs thématiques relatives à l'objet d'étude. Ce sont, pour la plupart, des notables, dont certains d'entre eux sont issus de familles de forgerons. Parallèlement à cette enquête, d'autres entrevues ont été menées auprès des dignitaires du village. Celles-ci réalisées par des Socioanthropologues, ont porté sur les aspects socio-économiques de la vie des populations de cette localité.

\section{La prospection archéologique}

Le groupe de recherche a mené une prospection intensive sur trois sites découverts lors des missions de 2010 à 2016. . Il s'agit des sites funéraires de Daovogo et de Nawavogo dominés par des sépultures et marqués par des pierres tombales en latérite taillées de différentes formes et du site d'habitat Nagakaha, ancien village de forgerons. Pour les deux premiers sites sus nommés, en dehors des sépultures, plusieurs tessons de céramiques ont été découverts en surface. Sur le site d'habitat Nagakaha, de nombreux restes de travail de fer, de la céramique et des sépultures isolées dont certaines à jarre ont été identifiés. 


\section{Relevés topographiques et fouille archéologique Les relevés topographiques}

Pour mieux cerner les sites au niveau de leur organisation spatiale et matérielle, nous avons procédé à des relevés topographiques. Ces opérations ont consisté dans un premier temps à délimiter les sites, ce qui a permis de les examiner dans tous leurs contours. Dans un deuxième temps, la répartition des sites en secteurs a été faite. Cette approche a permis de mieux identifier, inventorier les sépultures et les structures présentes. Dans cette perspective, les coordonnées géographiques de toutes les sépultures ont été relevées sur l'ensemble des sites. Celles-ci ont permis de localiser avec précision toutes les sépultures et les structures associées et de décrire l'organisation spatiale de ces sites funéraires. Cette démarche devrait permettre de géoréférencer tous les sites d'habitat, métallurgiques et funéraires identifiés. A long terme, ces données aideront à mieux localiser ces sites, afin de contribuer à leur protection et leur valorisation.

\section{La fouille archéologique}

Pour cerner le mode de vie des populations du passé de cette région, des fouilles archéologiques ont été réalisées sur certains sites. De ce fait, nous avons choisi les sites funéraires qui présentent une certaine spécificité au niveau de leur situation géographique, de leur organisation spatiale et de leur aspect en surface. Les sites d'habitat et de métallurgie du fer sont abordés dans deux thèses de doctorat unique impliquées dans ce projet ${ }^{6}$.

Sur le site funéraire de Nawavogo, deux fouilles ont été menées respectivement dans les zones de concentration, au niveau des secteurs 1 et 4 (Fig.1). Ces fouilles visent à comprendre les sépultures avec le mobilier associé s'il en existe. L'étude de cet ensemble devrait contribuer à une meilleure connaissance des peuples qui ont occupé ces espaces à travers l'approche socioanthropologique, archéologique, historique, paléoanthropologique et bioanthropologique.

$\mathrm{Au}$ niveau de la fouille archéologique la méthode horizontale et verticale ont été combinées. Au fur et à mesure que nous descendions en profondeur, des relevés et des plans étaient faits avec des photographies à l'appui. La sépulture du secteur 4 a livré un crâne d'enfant isolé avec des vestiges céramiques en surface et en contexte. La sépulture du secteur 1 quant à elle, a livré, après $2 \mathrm{~m}$ de profondeur, de nombreux artefacts céramiques mais pas de corps. Nous pensons ne pas avoir encore atteint la chambre sépulcrale qui pourrait contenir les restes humains. En effet, un document d'archives de 1939 écrit par l'administrateur Champeau, parle

${ }^{6}$ Les deux doctorants sont: Tui Lah Louis dont le sujet porte sur: «Les habitats protohistoriques de la région de la Bagoué » et Dapa Marie Joëlle qui travaille sur : «Les sites métallurgiques de la région de la Bagoué : le cas du Département de M’Bengué». 
d'un cimetière de la zone de Nimpurgué à Tingréla, sur une colline, comme celle de Nawavogo, qu'ils ont eu à visiter. Il affirme que «Les gens vulgaires sont enterrés dans de simples fosses, mais les chefs et les notables sont inhumés dans de véritables chambres funéraires creusées profondément dans le sol, 3 m environ, chambres auxquelles on accède par deux puits creusés à $2 m$ l'un de l'autre. " (Champeau, 1939: s. p.). Les analyses des restes humains se feront par les paléoanthropologues et les bioanthropologues de l'équipe. Celles-ci aideront à constituer une banque de données de la paléoépidémiologie et à comprendre les comportements écologiques, nutritionnels et sanitaires des populations passées et actuelles, afin de les intégrer dans les actions socio-sanitaires de développement de la région.

\section{Premiers résultats}

Pour cette première mission, des résultats importants sont obtenus.

\section{Sites funéraires}

L'intérêt de ces sites réside dans leur organisation spatiale, leur nature et les vestiges archéologiques en présence. Pour cette mission, deux sites ont été abordés. Ce sont les sites funéraires de Nawavogo et de Daovogo.

\section{Le site de Nawavogo}

Le site funéraire de Nawavogo qui signifie en langue locale "le campement de Nawa » est une nécropole localisée à environ 6 kilomètres à l'Ouest du campement éponyme. Il présente un relief accidenté. Localisé dans une forêt classée, au sous-bois clairsemé, ce site est une colline à deux versants séparée au sommet par un monticule qui longe le site dans le sens de la longueur, dans la direction Nord-Sud. De forme rectangulaire, le site s'étend sur une superficie de $30.720 \mathrm{~m}^{2}$, avec une longueur de $240 \mathrm{~m}$ et une largeur de $128 \mathrm{~m}$. Sur cette aire, plusieurs sépultures ont été identifiées, à proximité desquelles gisent des vases et des pots le plus souvent fragmentés. Nous avons identifié près de 45 \& sépultures inégalement réparties sur l'ensemble du site (Cf. fig. 1).

L'analyse topographique du site montre une forte concentration de sépultures dans les secteurs 1 et 4 qui occupent les versants Sud-Ouest et Nord-Ouest de la colline, abritant le site. Cependant, les secteurs 2 et 3 qui occupent les versants Sud-Est et Nord-Est du site n'abritent aucune tombe. Il est parsemé de blocs de latérites et la surface est dépourvue de vestiges céramiques à la différence du versant Ouest où l'on voit de prime à bord les vestiges céramiques sur une grande partie du site. 
Fig1 : Plan général du site de Nawavogo.

PLAN DU SITE DE NAWAVOGO (SITE FUNERAIRE)

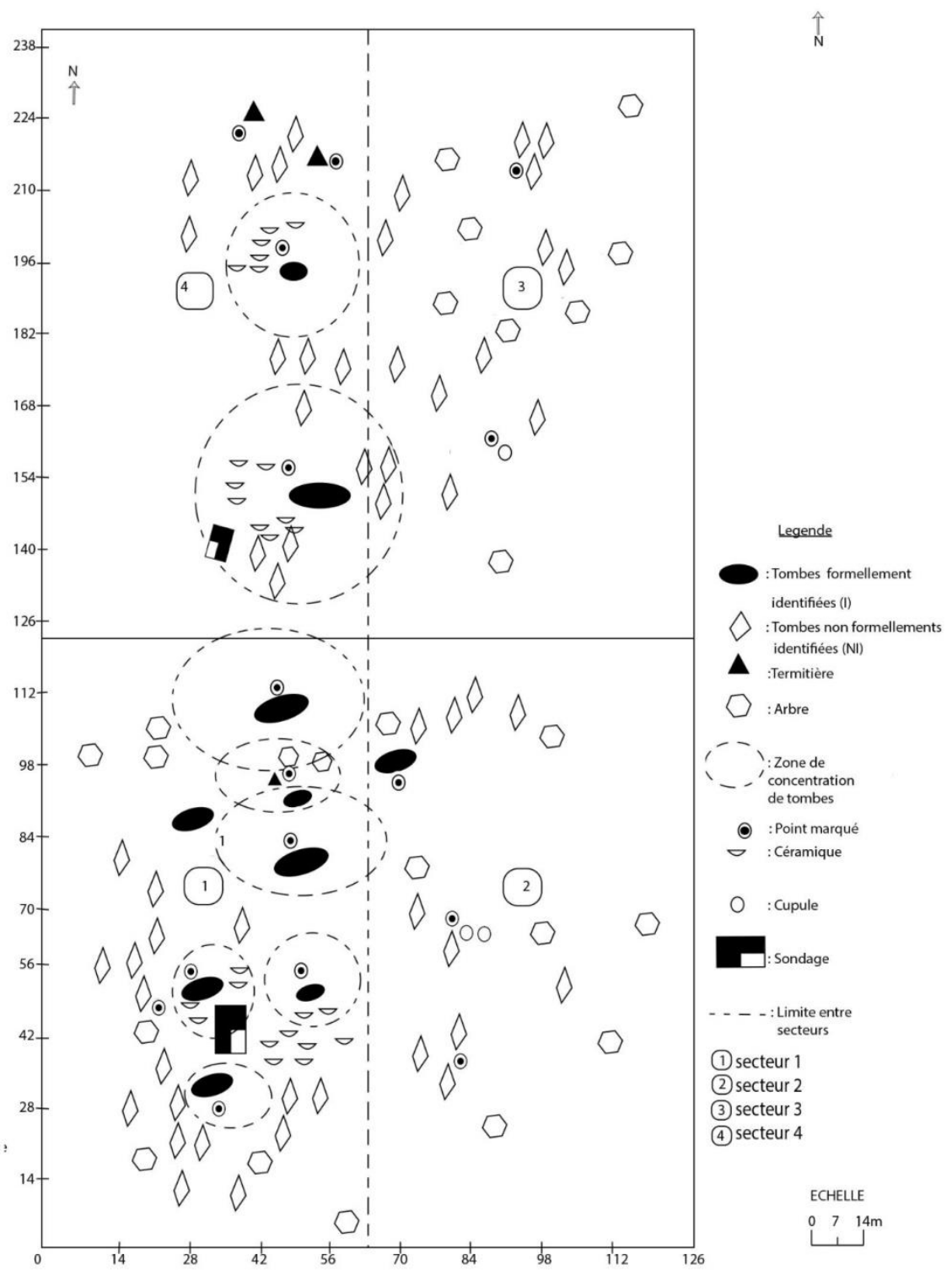

Ces sépultures sont ouvertes ou fermées. Les sépultures ouvertes sont facilement identifiables. Cette catégorie est bien présente dans les zones de concentration, au sommet et sur les flancs de la colline. Le nombre important sépultures ouvertes peut faire croire à un pillage du site funéraire. Cependant, une pratique ancienne propre aux populations de ces localités 
nous amène à prendre en compte cette donnée culturelle, sans négliger la piste de probables pillages. Maurice Delafosse l'explique bien dans la monographie du cercle de Korhogo de 1905. Il écrit ceci "Lorsque quelqu'un vient à mourir on fait venir toute la famille et on procède à l'enterrement (...). Si les parents résident très loin, on met le corps en terre, mais on a soin de ne fermer la tombe qu'au moyen d'une dalle de pierre que l'on soulève à chaque visite d'un membre de la famille; afin de déposer sur le mort les pagnes apportés par le visiteur. Une fois que tous les parents ont rempli ce devoir funéraire, on ferme définitivement la dalle et on la recouvre de terre. Certaines sépultures sont restées entrouvertes pendant plus de 10 ans, et j'ai vu procédé à l'enterrement définitif de gens décédés depuis près de 7 ou 8 ans, mais que les parents en voyage au loin n'avaient pas pu encore visité auparavant.»(Delafosse M, 1905 :22.). La distance qui sépare les sépultures n'excède généralement pas les deux mètres. En revanche, les sépultures fermées sont celles couvertes par une pierre tombale, à base de latérite taillée. Bien qu'elles soient fermées, ces sépultures laissent parfois entrevoir une ouverture sous la pierre tombale. Qu'elles soient ouvertes ou fermées, la céramique est présente en surface, le plus souvent en tesson de différentes tailles. Elle constitue un indicateur important de sépulture. En effet, sans la céramique en surface, on aurait cru être en présence d'une simple colline parsemée de blocs latéritiques (Photo 1).

Photo1 : Vue de la colline cuirassée sur laquelle se trouvent les sépultures.

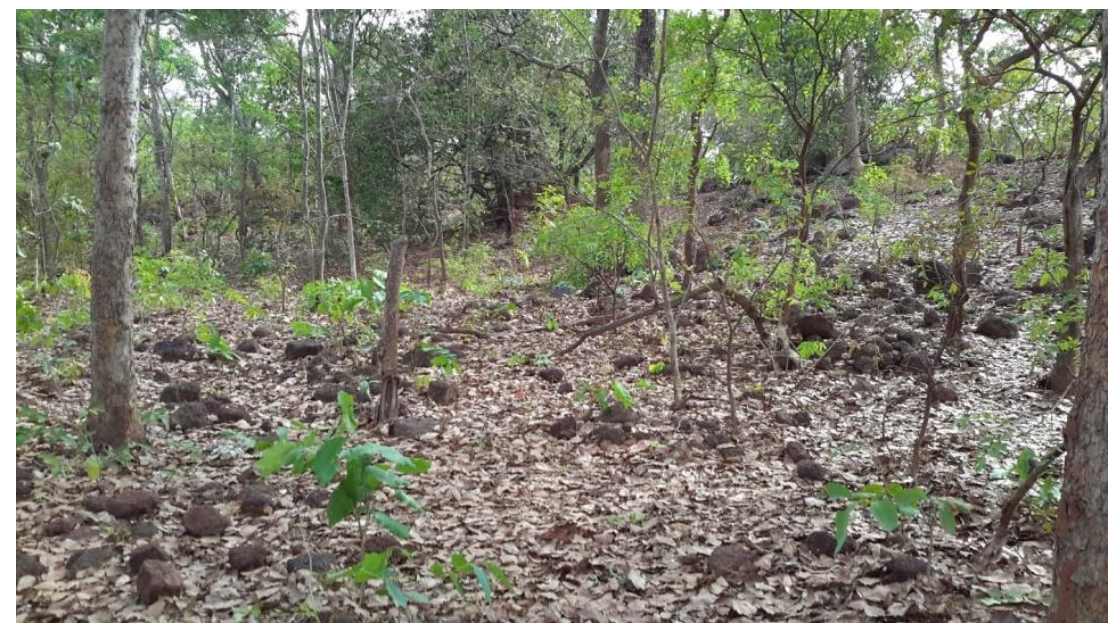

Photo Kienon-Kaboré T. Hélène

Pour une meilleure approche du site, il a été divisé en 4 secteurs. Les secteurs 1 et 4 , où ont été localisé un bon nombre de sépultures ont été fouillés.

Le secteur 1 est étendu sur un périmètre de $368 \mathrm{~m}$ et occupe le versant Sud-Ouest du site. Il regroupe à lui seul 35 sépultures inégalement 
réparties. Quatre points de concentration sont observés (Cf. fig.2). Le plus important dans la partie Sud-Est de ce secteur, couvre un diamètre d'environ $40 \mathrm{~m}$, avec un total de 18 sépultures formellement identifiées. Dans cette zone de forte concentration, les sépultures sont en majeure partie ouvertes et très rapprochées. Cette zone a été choisie pour poser le deuxième sondage. La céramique est également abondante avec des fragments de tailles variables. Le secteur 4 est aussi important en sépultures, mais moins dense que le secteur 1. Ce secteur regroupe 9 sépultures inégalement réparties. Une zone de forte concentration regroupant sept sépultures, a été relevée dans la partie Nord-Ouest du secteur (Cf. fig.3). Cet endroit où gisent des tessons de céramiques et des pots entiers, a été choisi pour poser le premier sondage, à l'emplacement d'une tombe. Les céramiques entières, de petites dimensions trouvées sur ce secteur sont soit posées sur le côté, soit complètement renversées, les fonds dirigés vers le haut. Les récipients céramiques renversés ont les bords complètement enfoncés dans le sol. Sur le site funéraire de Nawavogo, après observation de la surface, plusieurs types de pierres tombales et de dispositions peuvent être identifiés. Il s'agit des sépultures ouvertes, fermées, des pierres tombales de forme rectangulaire ou triangulaire ${ }^{7}$. Les sépultures fermées sont celles dont l'entrée est obstruée par une pierre tombale, soigneusement taillée dans la latérite, à la dimension de l'ouverture de la tombe. Qu'elles soient ouvertes ou fermées, elles sont en majeure partie concentrées dans la partie Ouest. Il existe néanmoins de rares sépultures isolées.

${ }^{7}$ Les formes des pierres tombales ne sont visibles qu'après exhumation. Lorsqu'on observe la surface du site, on ne voit que des pierres latéritiques de diverses formes enfouies dans le sol. 
Fig 2 : Plan du secteur 1 de l'ensemble funéraire de Nawavogo

PLAN DU SECTEUR 1 (Site Funéraire 1) NAWAVOGO

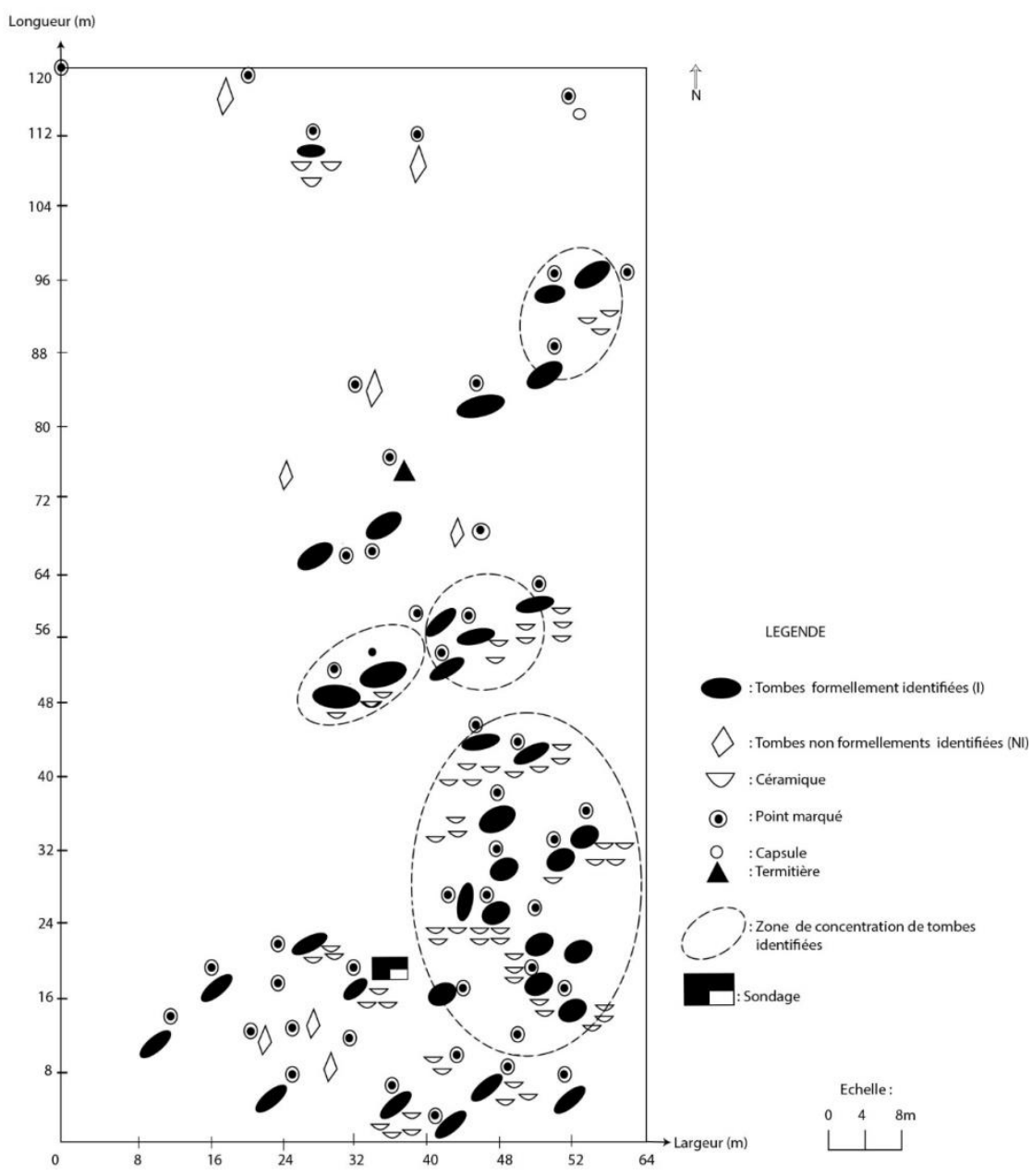


Fig3 : Plan du secteur 4 de sites funéraires l'ensemble funéraire de Nawavogo PLAN DU SECTEUR 4 (Site funéraire) NAWAVOGO

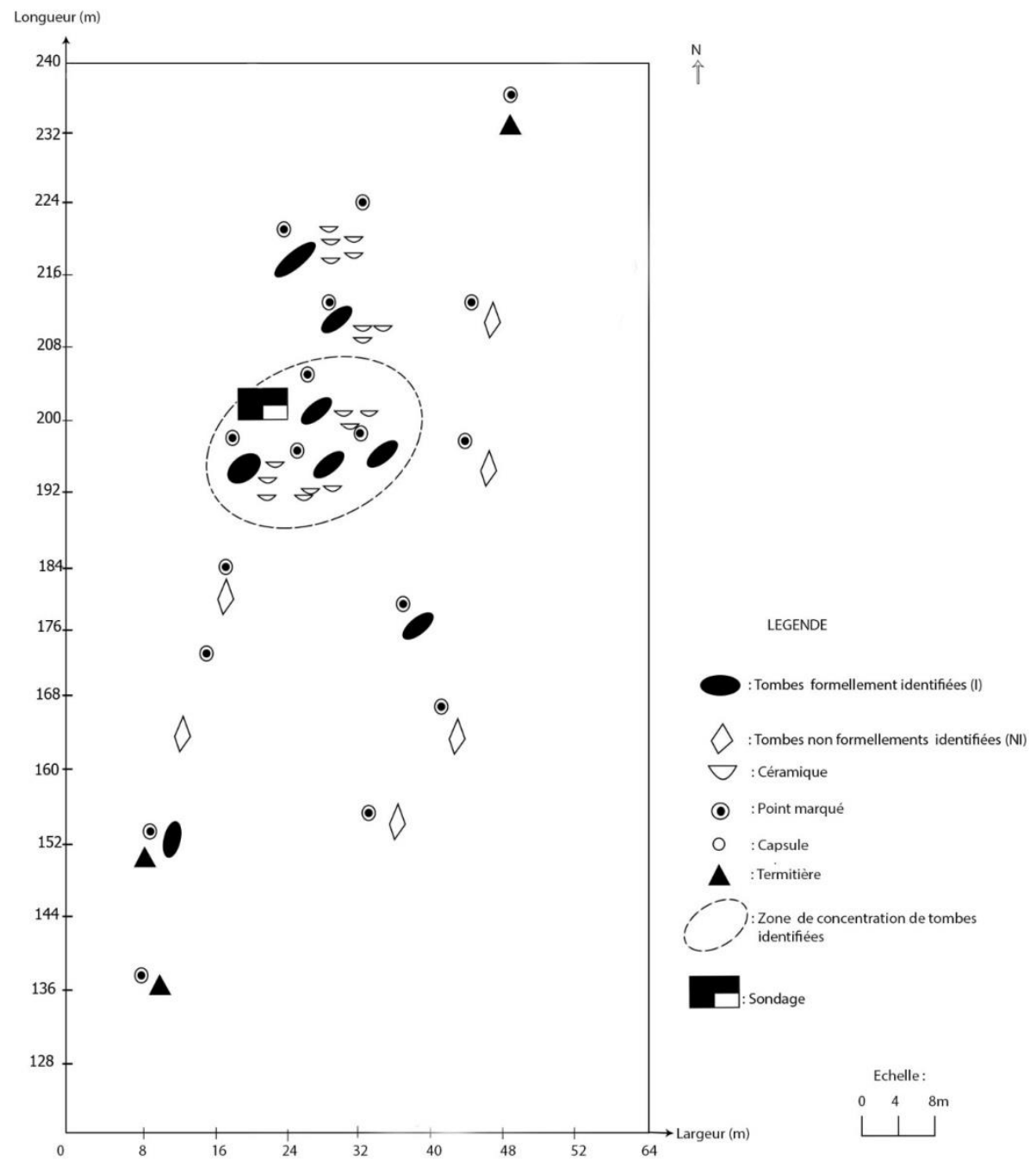


A côté de ces sépultures, facilement identifiables, il existe une autre catégorie de sépultures que nous avons nommées les sépultures non formellement identifiées. Pour ce type, il n'y a pas d'ouverture; les blocs latéritiques semblent ne pas être taillés. Toutefois, il est donné de constater la présence de tessons de céramiques à proximité des blocs. Outre les sépultures, des blocs latéritiques ont été identifiés sur toute l'étendue du site. $\mathrm{Au}$ vu de leur distribution et de leurs caractéristiques, deux types se dégagent dans l'ensemble. Il y a des blocs latéritiques naturels et des blocs anthropiques, taillés par l'homme. Les blocs naturels sont ceux qui d'apparence en observant en surface, n'ont subi aucune action de l'homme. Numériquement importants sur les secteurs 2 et 3 du site funéraire au versant Est, ils sont présents sur la quasi-totalité du site. A côté de ceux-ci, l'on note une catégorie de blocs latéritiques utilisés comme des pierres tombales, destinées à couvrir l'entrée principale de la tombe. A la différence des dalles latéritiques dites naturelles, les pierres tombales sont anthropiques; elles sont taillées, car portant des traces d'enlèvement souvent sur les deux faces. Dans cette dernière catégorie, deux formes ont été identifiées. Il s'agit des formes triangulaire et rectangulaire. (Photo 2). Notons que les pierres tombales ont été signalées dans d'autres parties de l'Afrique Sub-saharienne dont le Burkina-Faso. Des pierres tombales appelées Ya-kouga (Ya-kugri, au singulier) ont été identifiées sur une grande partie des sépultures des Tinguin- Biisi, les responsables de terres. Les pierres en granites ou en latérite de formes anthropomorphes associées à des figures géométriques représentent les têtes des défunts et donnent des informations sur la profession, la classe d'âge, la parenté, la situation et le statut social de ce dernier. Ces pierres tombales servent à identifiées des sépultures préalablement obstruées avec un couvercle en pierre. (Pacéré, T. 1990) 
Photo 2 : Pierre tombale latéritique de forme triangulaire avec les bords arrondis exhumée de la tombe 2 du secteur 1, laissant entrevoir l'ouverture de la sépulture qu'elle obstrue.

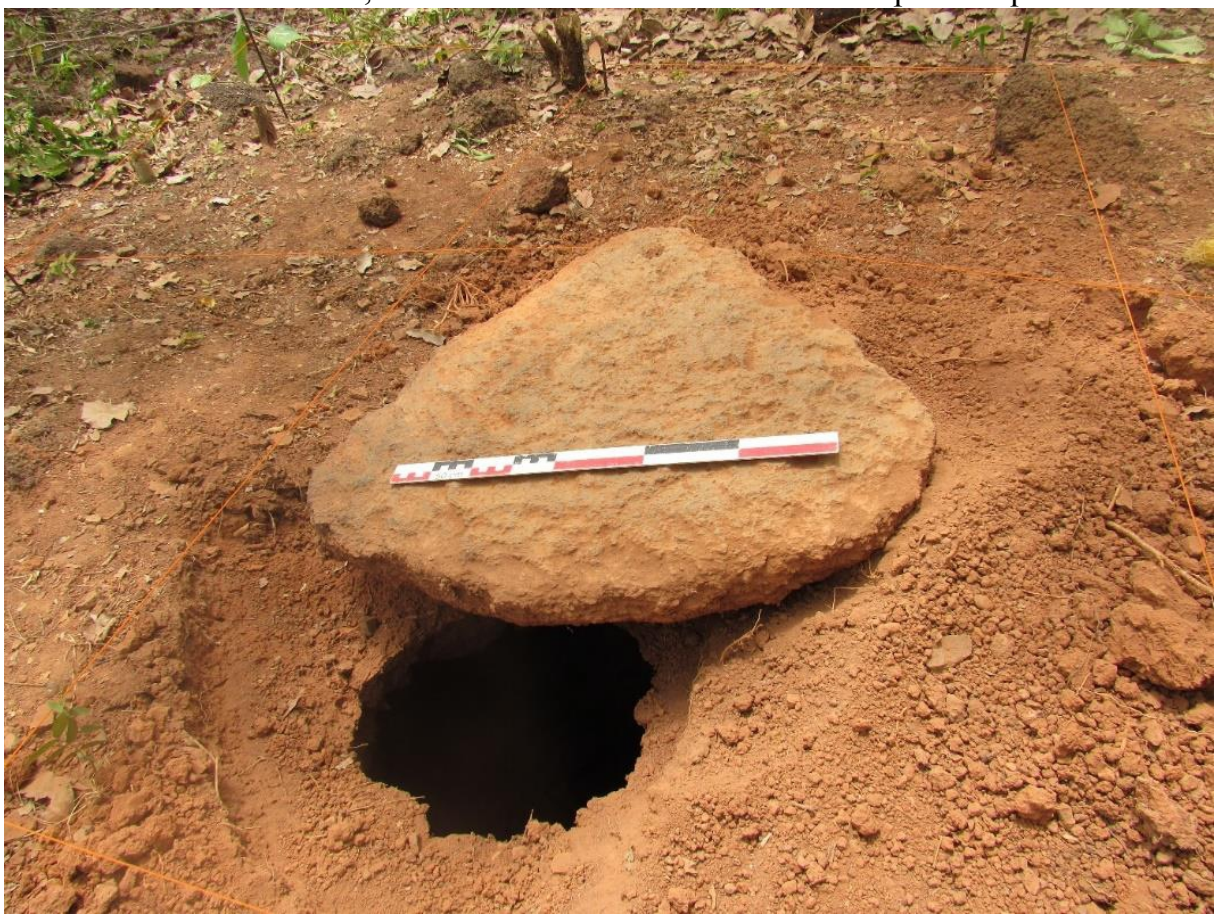

Photo : Kiénon-Kaboré T. Hélène

La céramique y est associée. Elle est en majeure partie fragmentée et bien localisée à proximité des sépultures. Elle constitue un indice important d'identification de sépultures. On l'aperçoit parfois sous la forme de céramique entière ou partiellement fragmentée. Il s'agit ici de la céramique initialement déposée sur les sépultures des défunts pour des rites funéraires. Nombre d'entre ces récipients sont des pots ou des jarres, de couleur rougeâtre et soigneusement décorés.

Sur l'ensemble du site, trois cupules creusées dans la cuirasse latéritique ont été enregistrées, dont l'une dans le secteur 3 et les deux autres côte à côte dans le secteur 2 . On ignore encore le rôle exact qu'elles ont pu jouer sur cet ensemble funéraire de Nawavogo.

\section{Le site de Daovogo}

Daovogo qui signifie en langue locale "le campement de Dao », est un site funéraire, situé à environ 5 kilomètres au Sud-Est du village de Poungbè. Il a été localisé à proximité du campement de Dao, sur un terrain nu entièrement labouré. A l'exception des buttes et des termitières qui parsèment la surface du site, c'est un vaste plateau de forme rectangulaire 
qui s'étend sur $172 \mathrm{~m}$ de long et $106 \mathrm{~m}$ de large. Quelques grands arbres surtout morts, se dressent dans la partie centrale du site.

Sur l'ensemble du site, 68 sépultures ont été identifiées, inégalement réparties. Trois points de concentration ont été enregistrés, le plus grand dans la direction Nord-Ouest, avec plus de 36 sépultures sur une étendue d'environ $60 \mathrm{~m}$. Les deux autres renferment 19 sépultures; elles ont été localisées dans la partie Sud-Ouest du site sur un diamètre total de $36 \mathrm{~m}$. Hormis ces zones de concentration, les autres sépultures sont complètement disséminées sur l'ensemble du site (Cf. fig. 4). Ce site se caractérise par des sépultures ouvertes. Quelques-unes d'entre elles se sont effondrées laissant apparaitre l'intérieur de la fosse fortement infectées par des abeilles et d'autres insectes. Certaines sépultures sont couvertes par des blocs de cuirasse latéritique taillés en forme triangulaire, quelque fois rectangulaire. Ces artefacts sont bien présents dans les zones de concentration des sépultures, mais elles ne figurent pas sur toutes les sépultures. Ces pratiques funéraires bien qu'elles ne soient pas suffisamment étudiées dans le cadre de cette étude, seraient des éléments importants pour l'histoire du peuplement de cette région du pays.

En dehors des sépultures, ce site livre aussi de la céramique en quantité importante. Ce sont des centaines de tessons de céramique fragmentés que nous avons découverts sur les aires de répartition de ces sépultures. Compte tenu de la récurrence des travaux champêtres, ce site est perturbé en surface. Ce qui explique la grande dispersion des tessons sur l'ensemble du site, parfois en dehors de l'emplacement des sépultures. Dans la direction Sud-Ouest, à proximité de deux sépultures, dans les amas de tessons de céramiques, deux bracelets métalliques ont été découverts.

Contrairement au site de Nawavogo, Daovogo dispose de quatre tumuli de tailles variables. Ces structures inhabituelles pour les sites de la zone, seraient anthropiques. La présence des tessons de céramiques en surface demeure un indice de leur caractère anthropique, mais pour l'heure nous ne disposons pas de données suffisantes sur ces structures. 
Fig. 4 : Plan général de l'ensemble funéraire de Daovogo

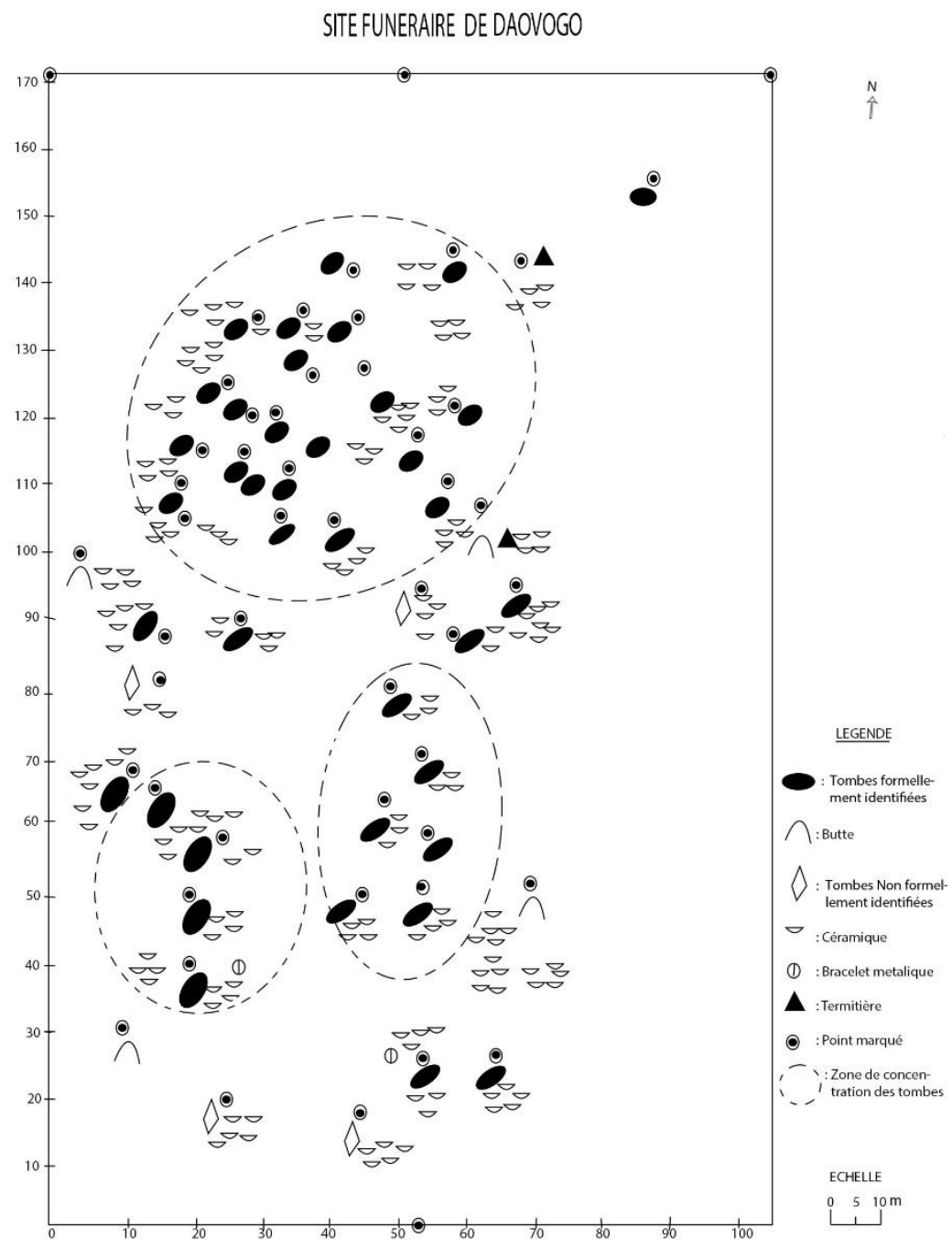

\section{Fouille de l'ensemble funéraire de Nawavogo}

Pour cette première mission, nous avons choisi le site de Nawavogo pour la fouille. Ce site est une nécropole située sur le flanc ouest d'une colline fortement cuirassée au sommet et allongée dans le sens Nord-Sud. Les sépultures sont identifiées à travers une forte concentration de fragments de céramique, la pose d'un bloc de pierre latéritique taillée ou la présence de fosse. Ces différents indices sont observés d'une part sur le flanc de la colline et d'autre part au sommet de celle-ci. Le sommet est marqué par des fosses en surface, creusée dans la cuirasse latéritique. Deux 
sépultures (sépulture 1 et sépultures 2) ont été fouillées sur le flanc Ouest de la colline.

\section{Description et fouille des sépultures 1 et 2}

\section{- $\quad$ Sépulture 1}

La sépulture 1 a été sélectionnée dans le secteur 4 situé au Nord du Flanc Ouest du site funéraire. Le corroyage a été posé sur un périmètre de $3 \mathrm{~m}$ sur 4 . Le choix de la tombe a été guidé par sa proximité avec un bloc de pierre latéritique taillé, la présence de trois céramiques entières disposées de façon renversées, les fonds vers le haut et l'organisation spatiale de l'ensemble du site. Le sondage a été posé dans le dernier carré du sondage dans la partie Sud, dans un rectangle de $1 \mathrm{~m}$ sur $3 \mathrm{~m}$.

De la surface jusqu'à $1 \mathrm{~m}$ de profondeur, le sol est resté rougeâtre avec une dernière couche très compacte. Toutefois, trois textures ont été observées au niveau du sol : une couche gravillonnaire avec de gros grains, une autre couche gravillonnaire avec des grains fins et un sol très compact. (Cf. fig. 5). Sur l'ensemble des décapages, des tessons de céramiques ont été mis au jour, ainsi qu'une boîte crânienne de petite taille, de couleur blanchâtre, à $-20 \mathrm{~cm}$ de profondeur. Au vu de sa taille, cette boîte crânienne pourrait appartenir à un enfant ; seules les analyses archéométriques permettront de la caractériser avec précision. Vue sa fragilité, il a été prélevé avec les sédiments et les mesures ne peuvent pas pour le moment être prises. Des termitières ont été observées sur une grande partie du sondage. Dans ce secteur, les pots de céramique sont de petites tailles. Ils sont différents de ceux répertoriés dans le secteur 1 qui sont en majorité un peu plus grands. Sur cette tombe, mis à part la pierre sépulcrale de forme rectangulaire, aucune cavité n'a été trouvée. On a l'impression qu'à cet endroit, les corps étaient posés à même le sol. A $1 \mathrm{~m}$ de profondeur, nous avons arrêté le sondage après avoir atteint le sol compact vierge. S'agit-il d'un secteur pour enterrer les enfants ? Existe-t-il une organisation spatiale spécifique du cimetière pour enterrer les morts ? Les petits pots trouvés sur ce secteur matérialisent ils des sépultures d'enfants ? Les traditionnistes affirment que dans les traditions anciennes, les enfants avaient un carré réservé et les adultes étaient également ensevelis selon le statut social du mort. Toutes ces questions pourraient avoir des réponses au cours des recherches futures. 
Fig. 5 : Coupe stratigraphique du profil Est de la sépulture 1

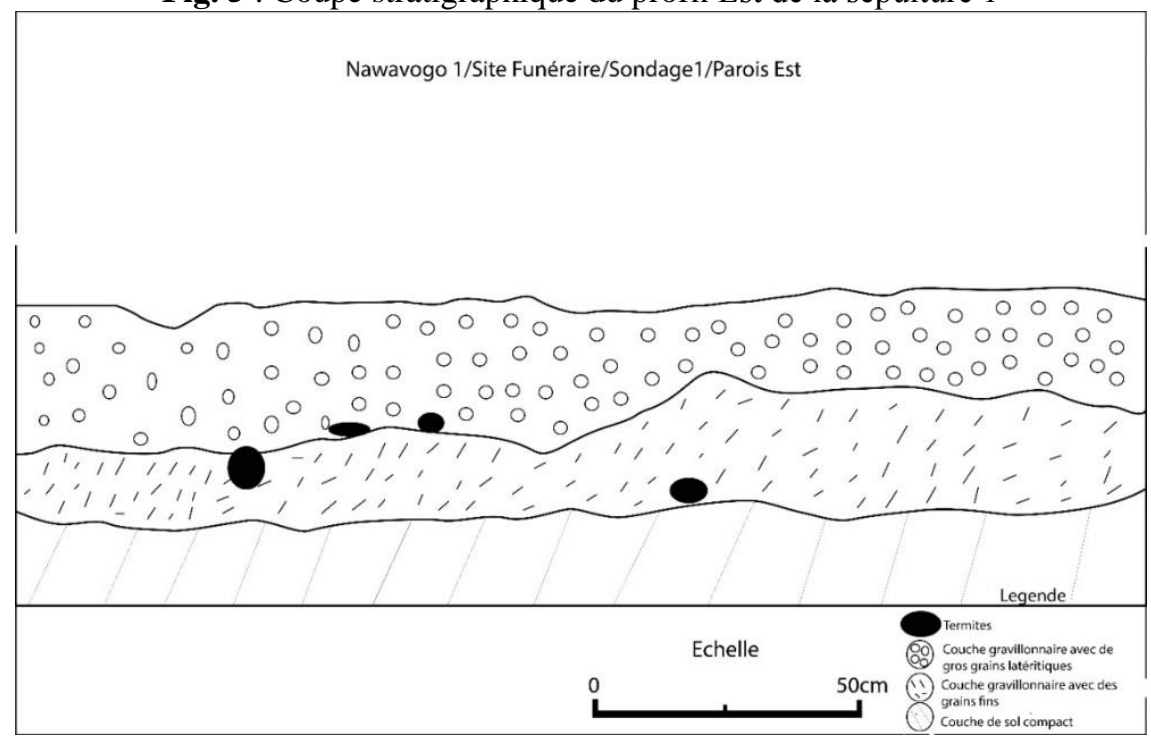

\section{- Sépulture 2}

La deuxième sépulture fouillée est située dans le secteur 1 du site, dans une zone à forte concentration de sépultures. Elle est marquée par une fosse située du côté Est et une pierre taillée, située à l'Ouest et distantes l'une de l'autre de $2 \mathrm{~m}$. L'orifice qui était fermé par une pierre latéritique du Côté Est s'est effondré laissant voir la pierre sépulcrale de forme rectangulaire à l'intérieur de la sépulture, tandis que celle de l'Ouest obstrue encore le deuxième orifice de la sépulture. La pierre sépulcrale en place et située du côté Ouest, façonnée dans la latérite est aplatie, taillée sur les deux faces et de forme triangulaire, arrondie sur les bords. Les mesures des trois côtés du triangle sont de $67 \mathrm{~cm}, 68 \mathrm{~cm}$ et $70 \mathrm{~cm}$ de long. L'orifice $(40 \mathrm{~cm}$ de diamètre) qu'elle laisse entrevoir en dessous après le déplacement de la pierre, est circulaire (Photo2). Le bord est bien régularisé avec des blocs de latérite et damé à l'intérieur avec des petits grains de latérite réguliers comme calibrés. La pierre sépulcrale en latérite dégagée laisse entrevoir un orifice d'une longueur dans le sens de la verticale de $170 \mathrm{~cm}$. La fosse est circulaire, rétrécie vers le haut et élargie à la base, donnant ainsi une forme tronconique avec un diamètre de près de $110 \mathrm{~cm}$. A partir de cette structure située à l'Ouest de la sépulture, s'allonge vers l'Est sur deux mètres de long une cavité dans laquelle les sédiments de surface se sont effondrés, rendant le sol meuble. Des fragments de céramique et des blocs latéritiques ont été prélevés tout au long du décapage qui a permis d'identifier trois couches. (Cf. fig. 7 et 8). Compte tenu du temps de la mission qui nous était imparti, la fouille s'est arrêtée à $2 \mathrm{~m}$ de profondeur au niveau d'une couche d'argile blanchâtre, sans atteindre la chambre funéraire. Les campagnes de fouilles 
ultérieures poursuivront les recherches afin de comprendre l'organisation de la sépulture. La description du cimetière et des sépultures de Nimpurgué en 1939 dans la zone de Tingréla par l'administrateur Champeau parle d'une profondeur de $3 \mathrm{~m}$ avant d'atteindre la chambre funéraire et de l'accès à la chambre sépulcrale par deux puits creusés à $2 \mathrm{~m}$ l'un de l'autre, comme ceux identifiés sur la sépulture 2 de Nawavogo. (Champeau, 1939 : s.p.)

Fig. 7 : Coupe stratigraphique du profil Sud-Est de la sépulture 2

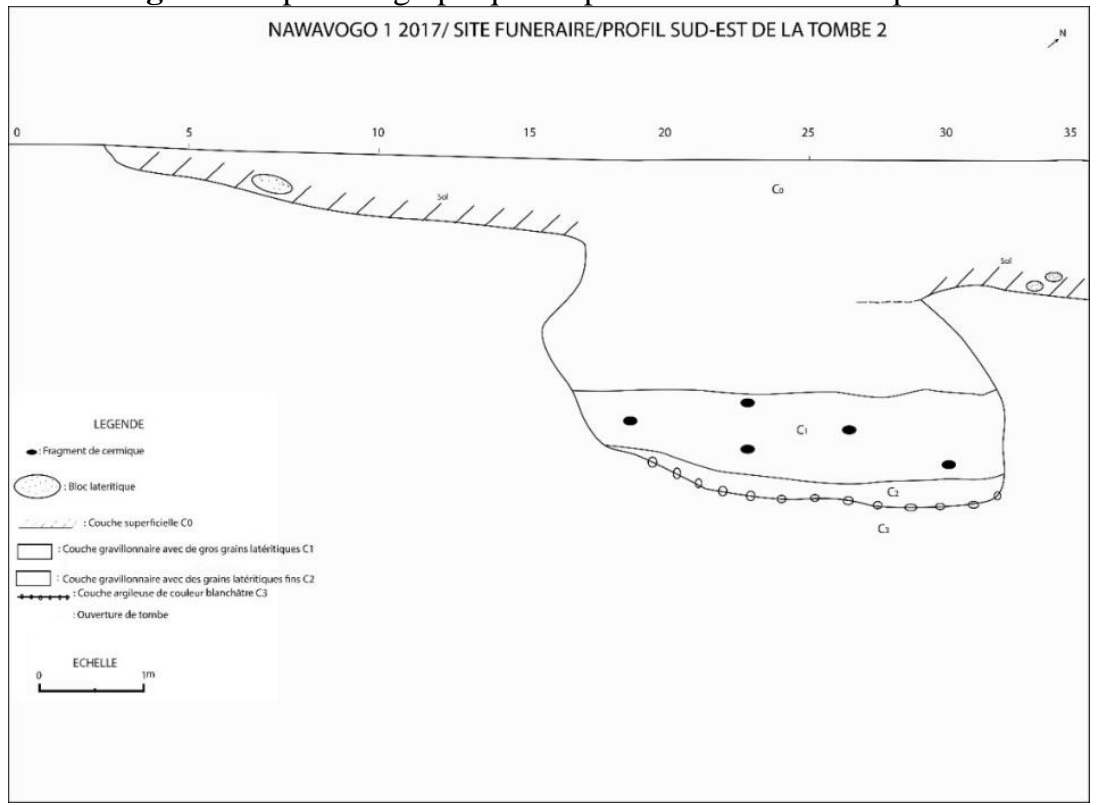

Fig. 8 : Profil Nord-Sud de la sépulture 2

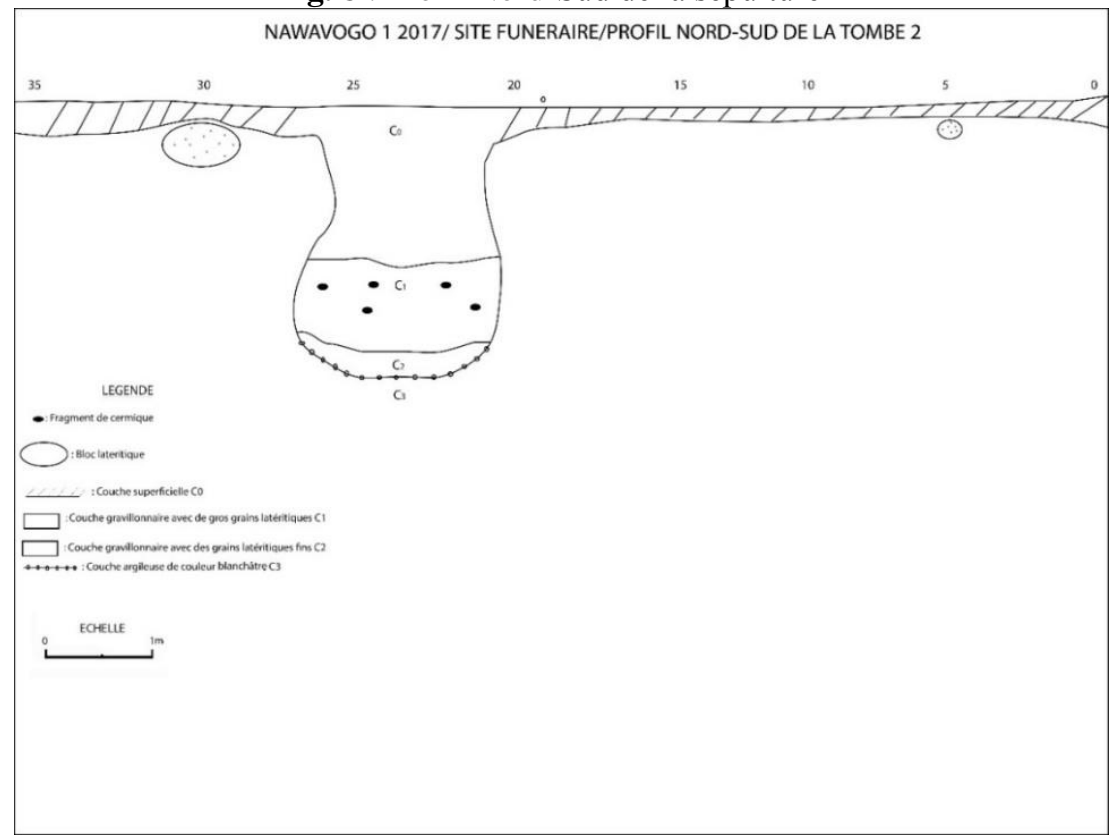


Les relevés topographiques, les premiers résultats des fouilles archéologiques et l'observation des sépultures en surface montrent bien qu'il y a une organisation spatiale bien définie. La situation géographique des sépultures, structures installées sur une colline et creusées dans la latérite, pour celle située au sommet de ce relief en élévation et l'obstruction des sépultures par des pierres latéritiques montre que les auteurs ont cherché une préservation des sépultures dans la longue durée. Les recherches sur les sépultures sont à leur début. Les résultats des investigations à venir dans une approche interdisciplinaire permettront de comprendre les sites et les cultures en présence.

\section{Conclusion}

La mission de recherche menée dans les localités de Poungbè et de Tongon fournit des résultats préliminaires importants. En effet, du point de vue spatial et des vestiges en présence, les sites funéraires de Nawavogo et de Daovogo se présentent comme deux sites essentiels pour une réelle étude interdisciplinaire.

Au niveau de la culture matérielle, l'on dispose d'une abondante céramique et d'une variété de sépultures. Dans le but de constituer un corpus céramique de la zone et de comprendre les séquences chrono-culturelles, des échantillons de fragments de céramiques ont été prélevés sur ces différents sites funéraires. Au total, 253 fragments de céramiques ont été collectés sur les deux ensembles funéraires. Une étude de la céramique est en cours et permettra de comprendre les caractéristiques des artefacts. Les céramiques des différentes nécropoles seront comparées entre elles au plan morphologique, des décors, des épaisseurs et des matières premières constitutives de ces céramiques afin de caractériser les techniques utilisées et les peuples auteurs de ces techniques anciennes. Les sites d'habitat ancien seront également abordés par l'étude de la céramique que l'on trouve afin de les comparer avec la céramique des sites funéraires.

L'étude permet déjà d'émettre des hypothèses et de donner des réponses préliminaires. Le choix du site de colline cuirassée à Nawavogo répond à la volonté des populations d'enterrer les morts sur des sites dont le matériau en pierre évoque la dureté, l'éternité. C'est peut être une volonté de garder les corps dans un environnement propice à leur protection dans la longue durée. Les populations de cette zone enterraient leurs morts du côté du soleil couchant. Ce qui justifierait la concentration des sépultures dans les secteurs 1 et 4, situés dans les directions Sud-Ouest et Nord-Ouest du site de Nawavogo. Au sujet des sépultures, à l'état actuel des investigations, deux traditions techniques sont identifiées. La première est un type de sépulture avec deux entrées ouvertes. La deuxième est une catégorie de sépultures présentant une seule entrée, fermée par une pierre tombale. Ces pratiques 
funéraires sont bien présentes sur les sites de Nawavogo et de Daovogo. Les sépultures sont pour la plupart regroupées. Toutefois, il existe quelques sépultures isolées. Ces pratiques funéraires devaient répondre à des préoccupations majeures, suivant la culture de ces populations.

Pour l'heure, nous ne disposons pas de données suffisantes pour une meilleure appréciation de ces sites. Les prochaines missions nous aiderons permettront de mieux traiter les questions de fond liées au mode de vie de ces populations et à leurs pratiques funéraires.

\section{References:}

\section{1-Sources orales}

\begin{tabular}{|c|c|c|c|c|}
\hline Nom et Prénom & $\begin{array}{c}\text { Date et lieu } \\
\text { de l'enquête } \\
\text { orale }\end{array}$ & Profession & Ages & observations \\
\hline $\begin{array}{c}\text { Ouattara Gniré } \\
\text { Zié } \\
\text { Souleymane }\end{array}$ & $\begin{array}{l}\text { Nawavogo le } \\
17 \text { au } 20 \text { Juin } \\
2011 \text { et du } 11 \\
\text { au } 15 \text { Mars } \\
2014\end{array}$ & $\begin{array}{c}\text { Cultivateur/ chasseur } \\
\text { Dozo }\end{array}$ & 60 ans & $\begin{array}{l}\text { Notre guide dans } \\
\text { la brousse. } \\
\text { Connaissances } \\
\text { profondes des } \\
\text { plantes et des } \\
\text { traditions }\end{array}$ \\
\hline Sekongo Drissa & $\begin{array}{c}\text { Poungbè le } 21 \\
\text { et } 22 \text { Juin } \\
20112012\end{array}$ & Cultivateur & 55 ans & \\
\hline Tuo Zana & $\begin{array}{c}\text { Poungbè le } 21 \\
\text { et } 22 \text { Juin } \\
20112012\end{array}$ & Cultivateur/forgeron & 54 ans & \\
\hline $\begin{array}{l}\text { Coulibaly } \\
\text { Dossongui }\end{array}$ & $\begin{array}{c}\text { Poungbè, } 18 \\
\text { et } 19 \text { Mars } \\
2017\end{array}$ & Cultivateur/ Notable & 65 ans & $\begin{array}{l}\text { Enquête en } \\
\text { groupe }\end{array}$ \\
\hline $\begin{array}{l}\text { Coulibaly } \\
\text { Nitchanfou }\end{array}$ & $\begin{array}{c}\text { Poungbè, } 18 \\
\text { et } 19 \text { Mars } \\
2017\end{array}$ & Cultivateur Notable & 70 ans & $\begin{array}{l}\text { Enquête en } \\
\text { groupe }\end{array}$ \\
\hline $\begin{array}{l}\text { Coulibaly } \\
\text { Sogofolo }\end{array}$ & $\begin{array}{c}\text { Poungbè, } 18 \\
\text { et } 19 \text { Mars } \\
2017\end{array}$ & Cultivateur/ Notable & 70 ans & $\begin{array}{l}\text { Enquête en } \\
\text { groupe }\end{array}$ \\
\hline $\begin{array}{l}\text { Coulibaly } \\
\text { N'Golo }\end{array}$ & $\begin{array}{c}\text { Poungbè, } 18 \\
\text { et } 19 \text { Mars } \\
2017\end{array}$ & $\begin{array}{c}\text { Cultivateur/ } \\
\text { Forgeron/Notable }\end{array}$ & 75 ans & $\begin{array}{l}\text { Enquête en } \\
\text { groupe }\end{array}$ \\
\hline Soro Fatogoma & $\begin{array}{c}\text { Poungbè, } 18 \\
\text { et } 19 \text { Mars } \\
2017\end{array}$ & Cultivateur & 60 ans & $\begin{array}{l}\text { Enquête en } \\
\text { groupe }\end{array}$ \\
\hline Tuyo Bembara & $\begin{array}{c}\text { Poungbè, le } \\
18 \text { et } 19 \text { Mars } \\
2017\end{array}$ & $\begin{array}{l}\text { Sécrétaire du comité de } \\
\text { gestion du village }\end{array}$ & 37 ans & $\begin{array}{l}\text { Enquête en } \\
\text { groupe }\end{array}$ \\
\hline Yéo N'Golo & $\begin{array}{c}\text { Poungbè, le } \\
18 \text { et } 19 \text { Mars } \\
2017\end{array}$ & Cultivateur/ Notable & 73 ans & $\begin{array}{l}\text { Enquête en } \\
\text { groupe }\end{array}$ \\
\hline Tuyo Penan & Poungbè le 24 & Cultivateur/ Forgeron & 68 ans & Enquête \\
\hline
\end{tabular}


1. CHAMPEAU, 1939, Découverte d'un ancien cimetière à mi-chemin sur la route de Kebi à Nimpurgué, Archives Nationales de Côte d'Ivoire, S.P.

2. DELAFOSSE M. 1905, Essai de monographie du cercle de Korhogo. Région de Kong. (Côte d'Ivoire), Archives nationales de Côte d'Ivoire, P22

3. AVY Stéphane, Koffi. et Al, 2016, Cartographie de l'état du couvert végétal du nord de la Côte d'Ivoire à partir d'images satellites: Exemple de la zone de Korhogo, European Scientific Journal October, Edition vol.12. PP.204-2013

4. BEAUDOU Alain., SAYOL R. 1980, Etude de la région BoundialiKorhogo (Côte d'Ivoire), Cartographie et typologie sommaire des sols, Feuille BOUDIALI Feuille KORHOGO, Notice explicative No 84 , office de la recherche scientifique et technique outre-mer, Paris, $58 \mathrm{p}$.

5. DUC Philippe, 2012, Forgerons de la région de Korhogo (Côte d'Ivoire) essai en anthropologie des techniques. Mémoire de master en ethnologie, Institut d'ethnologie, Université de Neuchâtel, 2012. $91 \mathrm{p}$

6. GONNIN Gilbert. et ALLOU Kouamé René, 2006, Côte d'Ivoire : les premiers habitants. Histoire de la Côte d'Ivoire, les Editions CERAP, 121p

7. ISSENMANN Régis et Al, 2013, L'occupation funéraire au début du second âge du fer dans le sud-châlonnais autour de la tombe aristocratique de Moncetz-Longevas la commune (marne) Revue Archéologique de l'Est, t. 62-2013, p. 53-88 (C) SAE 2013

8. KIENON-KABORE Timpoko Hélène, 2012, La sidérurgie directe en Côte d'Ivoire: Contexte et résultats de la recherche. European Scientific Journal Vol 8, PP.144-161

9. KIENON-KABORE Timpoko Hélène, 2010, Prospection archéologique dans la région de Toumodi et de Korhogo (Côte d'Ivoire), SLSA Jahresbericht, PP 42-54.

10. PACERE Titinga, 1990, Les Ya-Kouga ou pierres tombales du Burkina. Collection «Le musée expliqué » 